Journal of Innovative Image Processing (JIIP) (2021)

Vol.03/ No. 01

Pages: 7-20

https://www.irojournals.com/iroiip/

DOI: https://doi.org/10.36548/jiip.2021.1.002

\title{
Analysis of Adaptive Image Retrieval by Transition Kalman Filter Approach based on Intensity Parameter
}

\author{
Dr. R. Dhaya, \\ Department of Computer Science, \\ Sarat Abida Campus -King Khalid University, \\ KSA. \\ dhayavel2005@gmail.com
}

Abstract- The information changes in image pixel of retrieved records is very common in image process. The image content extraction is containing many parameters to reconstruct the image again for access the information. The intensity level, edge parameters are important parameter to reconstruct the image. The filtering techniques used to retrieve the image from query images. In this research article, the adaptive function kalman filter function performs for image retrieval to get better accuracy and high reliable compared to previous existing method includes Content Based Image Retrieval (CBIR). The kalman filter is incorporated with adaptive feature extraction for transition framework in the fine tuning of kalman gain. The feature vector database analysis provides transparent to choose the images in retrieval function from query images dataset for higher retrieval rate. The virtual connection is activated once in single process for improving reliability of the practice. Besides, this research article encompasses the adaptive updating prediction function in the estimation process. Our proposed framework construct with adaptive state transition Kalman filtering technique to improve retrieval rate. Finally, we achieved $96.2 \%$ of retrieval rate in the image retrieval process. We compare the performance measure such as accuracy, reliability and computation time of the process with existing methods.

Keywords: Image Retrieval, Kalman filter

ISSN: 2582-4252 (online)

Submitted: 26.12 .2020

Revised: 30.01 .2021

Accepted: 24.02.2021

Published: 09.03.2021 
Journal of Innovative Image Processing (JIIP) (2021)

Vol.03/ No. 01

Pages: 7-20

https://www.irojournals.com/iroiip/

DOI: https://doi.org/10.36548/jiip.2021.1.002

\section{INTRODUCTION}

Recently, the visual based information is used to search many inquiries in the communication internet protocol. It is a fast well growing technology in the search engine based on the image and video [1][2]. The researchers are concentrating this maintaining of database for both contents. This content based image retrieval system is defining the many features of images for various database. This application is extended up-to retrieving the similar images or video [3][4]. This system can be used in many numerous field such as medical image processing, commercial advertising, scientific patent database management system, military application and all authorized database system [5]. This type of classification or retrieval the images are one of the challenging task in huge amount of database currently in an internet domain. This effective finding way consisting of analyzing, make table, describing, classifying, identifying, similarity measures in a row $[6][7][8]$.
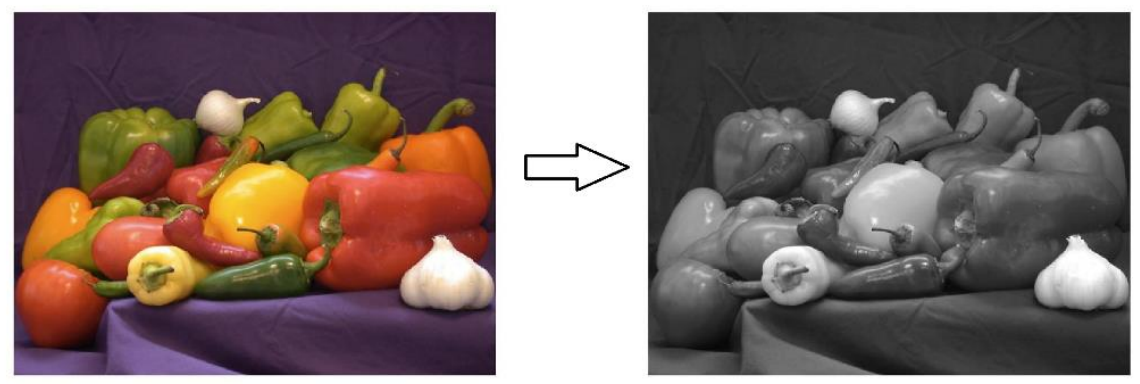

Figure 1 Example of RGB image to Grayscale

Based on the image detail there will be many applications such as weather forecasting and prediction of tsunami and any other disaster. But all algorithm is focusing to recover the image very speedily with more accurately [9][10]. The primary visual contents are used in the system to retrieve the image with more accuracy. Based on the intensity variations are influencing in an images commonly. The edges and intensity information are extracted from the images and computing the histogram distribution of the images has to match for similarities between them [11][12]. The large scale collections are needed to check this similarities of images between them currently for high accuracy. Earlier, the computational visual attention provides the details for retrieval function to match the similarities. Later, the additional features are added to extract the feature from the images for better retrieval model. Many research papers analysis content based 
Journal of Innovative Image Processing (JIIP) (2021)

Vol.03/ No. 01

Pages: 7-20

https://www.irojournals.com/iroiip/

DOI: https://doi.org/10.36548/jiip.2021.1.002

image retrieval method to recover the data effectively [13][9]. This method is used to extract the color spaces from distinguish the texture and spatial pattern. Figure 1 is showing example of RGB to grayscale image.

\section{ORGANIZATION OF THE RESEARCH}

The structure of the research article is organized as follows; Section 3 gives recent research techniques for images retrieval algorithm. Section 4 discusses the complication of the implementation of kalman filtering approach in an adaptive image retrieval proposing a solution to the process for future trends. Section 5 delivers a comparison of various method in image retrieval procedure. The conclusion and the future task discusses in section 6 .

\section{PRELIMINARIES}

The past research articles consist of many content based image retrieval in image process analysis. The feature extraction in images performs by wavelet transform; this technique suffers from many extraction problems from the image co-ordinal axis. The wavelet transform is constructing based on many filter such as analysis and synthesis filter problem. Therefore, many approaches are proposed to overcome those problems of implementation in wavelet transform [14]. Sathesh et al proposed the concept of dual tree wavelet construction for denoising images [15]. Kokare et al proposed the concept of construction of wavelet filter is derived based on dimensional and complex wavelet filter [16]. The prediction algorithm used to predict the website image search scenario was proposed by Nie et al [17]. They complete their proposed model with adaptive graph theory for image position in web page. Nie et al introduce the heterogeneous probability network estimation for the images with many questioners. They believed layer level construction techniques for image retrieval. The identification of many unreliable webpages from the internet sources can be categorized by this level based image ranking target. The heuristic approach is introduced to predict the relevance score of images in the pages through complex domain based concept [18].

There are many feature extraction methods exist based on the intensity of images. The colour histogram is mostly used in many feature extraction approach in complex model. But is very reliable and efficient still. Based on histogram distribution, the spatial time domain is 
Journal of Innovative Image Processing (JIIP) (2021)

Vol.03/ No. 01

Pages: $7-20$

https://www.irojournals.com/iroiip/

DOI: https://doi.org/10.36548/jiip.2021.1.002

changing to frequency domain concept fails to recover the original image content. Therefore, correlogram and coherence vector type approach is efficient for specific spatial structure [19][20]. The texture construction is performing with many filters such as wavelet and Gabor for image reconstruction. The image description is calculated by many features like shape, intensity, curvature, angular function, orientation transforms like steerable wavelets. But these techniques are facing executing time, lack of accuracy, unreliable problems. And it includes many process to predict and reconstruct the polynomial factors for image retrieval approach [21][22][23]. These problem can be minimized with rotation based phase invariance in an image intensity. This invariant feature is locating certain point with achieving scale function and rotation invariance property of image at different scale space.

The SURF and PCA-SIFT method is proposed to minimize the complexity of the feature extraction problems above mentioned [16]. Harris et al proposed technique is contained steerable function for the feature extraction of local image features by prediction model. The image retrieval is performed by dictionary based similarity match at the tail of the process in an image retrieval. Here large number image dataset provides better accuracy in the process. The multi feature fusion technique is computing the edge property of the image classification. The color and texture of the image can be combined by the structure element descriptor for elements steerable function. Lie et al proposed the method to integrate the color and feature extraction for image retrieval [24]. The deep learning approach provides answer for many problems arises by filtering techniques for image retrieval. Because of pre-trained parameter is used to extract local or edge features from the original images by many layer function for example Convolutional Neural Network (CNN) [25]. The network architectures in deep learning approach can give good retrieval rate and recovering rate by lots of trained the data. Since it is a knowledge based technique and complex process for image retrieval.

\section{RESEARCH GAP}

But still, the handling of adjacent frames is inefficient and utilizing low in many features extraction. Therefore, the observation and utilization can be improving by state transition approach by kalman filtering technique. This approach includes the parameter for estimation and error 
Journal of Innovative Image Processing (JIIP) (2021)

Vol.03/ No. 01

Pages: 7-20

https://www.irojournals.com/iroiip/

DOI: https://doi.org/10.36548/jiip.2021.1.002

correlation function to reduce those complexities that mentioned above. The proposed model can be predicted estimation factor for intensity of the images automatically without any adjustment of camera calibration. Adaptive kalman filtering approach facilitates the automatic and accurate retrieval of images with good intensity.

\section{METHODOLOGIES}

The images are represented as color through histogram distribution that is showed in figure 1. The features are extracted from given RGB color image for the pixel information based on the histogram function of it [26].

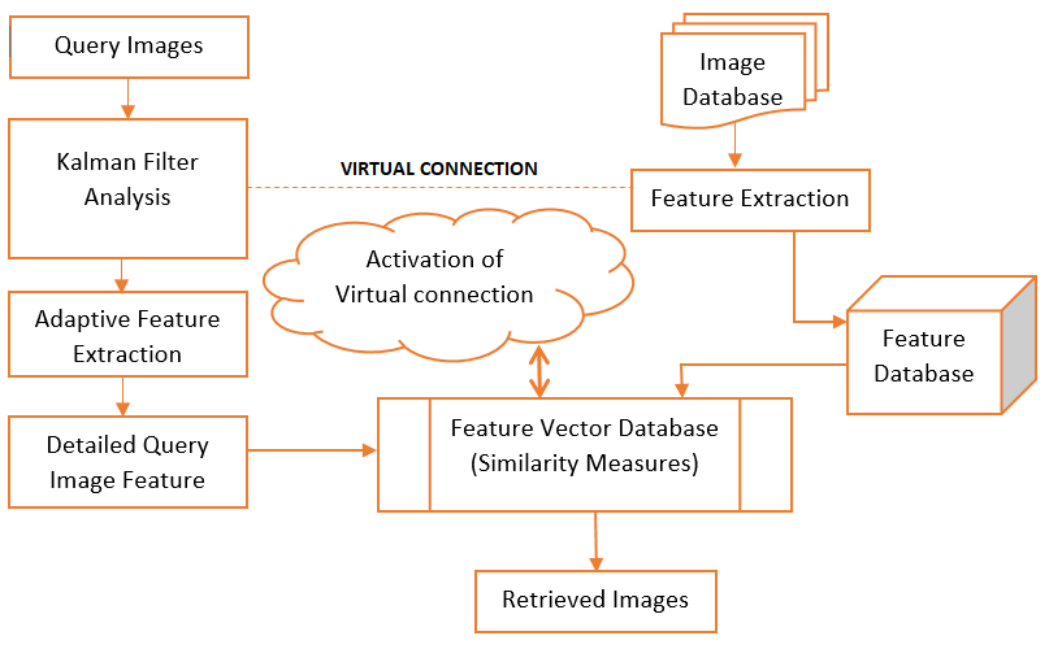

Figure 2 Workflow of Proposed Framework

The proposed framework is showing in the figure 2. Get the query image to analysis by our prosed method with virtual connection of image database. The state transition for kalman filter shows in figure 3 . The proposing model can be derived and stepped here as follows,

\section{Step 1:}

The image representation is denoted as $x, y$ for $R, G, B$. Due to this representation, the extra features can extract from the image such as intensity, edge, orientation features. The intensity feature map can be computed as these three intensity values for the various angular orientation in

ISSN: 2582-4252 (online) 
Journal of Innovative Image Processing (JIIP) (2021)

Vol.03/ No. 01

Pages: 7-20

https://www.irojournals.com/iroiip/

DOI: https://doi.org/10.36548/jiip.2021.1.002

the images [27] [28]. This output is combined with spatial information of the given image based on local region of interest features by the estimated values.

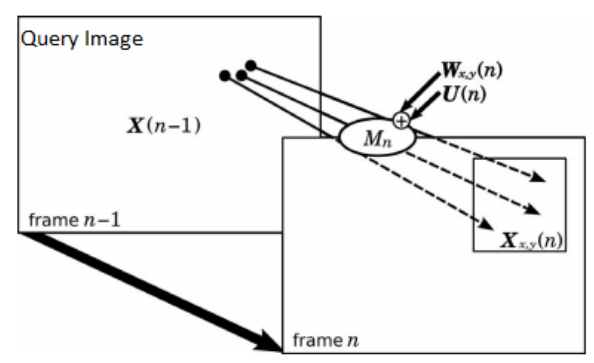

Figure 3 Proposed state transition framework

\section{Step 2:}

The visual feature extraction is very sensitive in the images due to changes of content and texture structure. The color and edge of the image should be handled very sensitive with extracted visual features relevantly by estimation factor.

$$
\bar{X}_{x, y}(n)=M_{n}(\hat{X}(n-1))+W_{x, y}(n)
$$

\section{Step 3:}

The kalman filter approach is used to estimate the state space with discrete transform process by difference equation for state transition model [29][30]. The time update is changing for every estimation prediction and correction by predict the state space, Initially, $\mathrm{k}=0$ for the estimation, Let's take,

$$
\hat{x}_{k}^{-}=A \hat{x}_{k-1}+B u_{k}
$$

\section{Step 4:}

This filter approach is measuring noise variance that produce random values in the images leads to nonlinear state. Generally, the image is computing through spatial time values by state space matrix model. Predicting with error covariance,

$$
P_{k}^{-}=A P_{k-1} A^{T}+Q
$$

\section{Step 5:}

The estimation error can be tuned by kalman filter state space gain parameter as given in the equation below; Computing Kalman gain, 
Journal of Innovative Image Processing (JIIP) (2021)

Vol.03/ No. 01

Pages: 7-20

https://www.irojournals.com/iroiip/

DOI: https://doi.org/10.36548/jiip.2021.1.002

$$
K_{g}=P_{k}^{-} H^{T}\left(H P_{k}^{-} H^{T}+R\right)^{-1}
$$

Adaptive update with minimum estimation by $Z_{k}$

$$
\hat{x}_{k}=\hat{x}_{k}^{-}+k_{g}\left(z_{k}-H \hat{x}_{k}^{-}\right)
$$

Where $\mathrm{T}$ denotes the state variable at time $\mathrm{k}$.

The random noise is considering for this retrieval process and current pixel can be tuned; the effective image retrieval is possible [31][32][33]. The adaptive function incorporates the obtained state transition model for the observation as follows;

$$
P_{x, y}(n)=M_{n}^{e}\left(P_{a}(n-1)\right)+Q_{U}(n)
$$

The diagonal matrices are comprising the data of diagonal elements in the structured image. The $M_{n}^{e}$ is contained covariance matrix with previous slide in the image based on the estimation movement [34][35][36]. The kalman filter gain has been adjusted based on the motion of estimation as follows;

$$
K_{g_{x, y}}(n)=P_{x, y}(n) H_{x, y}^{T}(n)\left[H_{x, y}(n) P_{x, y}(n) H_{x, y}^{T}(n)+Q_{V}(n)\right]
$$

\section{Step 6:}

The iteration can be increasing by changing the $\beta$ factor in the each state transition as denoted as,

$$
a K_{x, y}^{-\beta d} \leftarrow \frac{\left\|G_{x, y}(n)-A(n)\right\|}{\left[G_{x, y}(n)-A(n)\right]^{T}\left[\hat{X}_{x, y}-A(n)\right]}
$$

The $G_{x, y}(n)$ is diagonal matrix elements. This adaptive function gain for kalman filtering approach is providing good estimation of local feature extraction of the image. Also the prediction parameter $\hat{X}_{x, y}(n)$ is given as follows;

$$
\hat{X}_{x, y}(n)=\bar{X}_{x, y}(n)+K_{x, y}(n)\left[Z_{x, y}(n)-\left(H_{x, y}(n) \bar{X}_{x, y}(n)+F_{x, y}(n)\right)\right]
$$

Finally, we got derived the adaptive function prediction parameter for the image retrieval is given as,

$$
a P_{x, y}(n)=P_{x, y}(n)-K_{x, y}(n) H_{x, y}(n) P_{x, y}(n)
$$

\section{RESULTS DISCUSSION}

Making the histogram distribution is linear that provides good experimental result for image retrieval which is shown in figure 4. 
Journal of Innovative Image Processing (JIIP) (2021)

Vol.03/ No. 01

Pages: 7-20

https://www.irojournals.com/iroiip/

DOI: https://doi.org/10.36548/jiip.2021.1.002

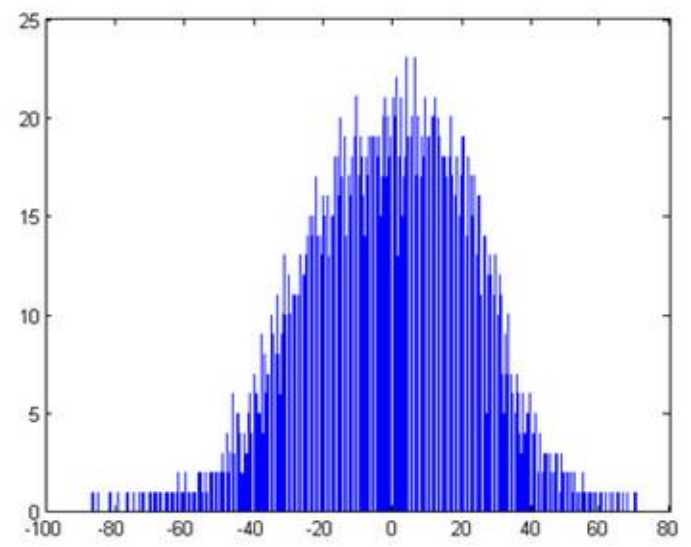

Figure 4 Histogram distribution of image representation

Figure 5 shows input image representation of RGB panel. The region of interest values is detected location direction of edges in perfect way to obtain the intensity variation [37]. The neighborhood pixel is carried the depth intensity value of various layer function with different angular motion. This visual cortex is choosing the orientation of angular motion estimation parameter. This certain function is taking the input of the given image that better texture accuracy for local extraction [38][39][40]. Correctness of the local update measurement data,

$$
P_{l k}=\left(I-K_{g} H\right) P_{k}^{-}
$$

Here the multiresolution analysis takes place to assemble the images by filter approach with various standard deviations [41][42]. This approach computation is preferred better reconstruction for feature vector of the given image intensity.
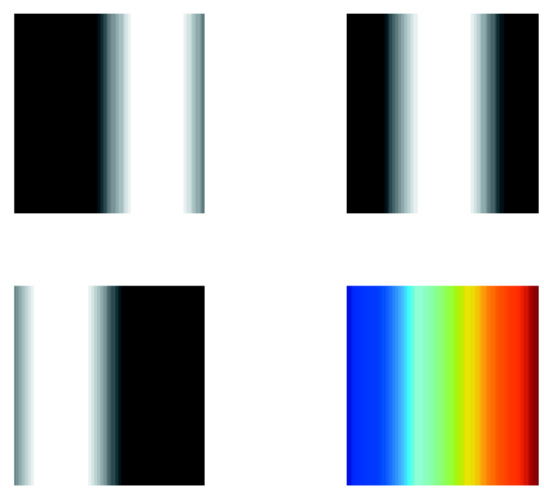

Figure 5 RGB panel

ISSN: 2582-4252 (online) 
Journal of Innovative Image Processing (JIIP) (2021)

Vol.03/ No. 01

Pages: 7-20

https://www.irojournals.com/iroiip/

DOI: https://doi.org/10.36548/jiip.2021.1.002

Our proposed method consists of four state space model to estimate for edges, intensity, distance of features and an angular motion. These combined approach is showing good result of image retrieval process with the help of weighted approach. Finally, our proposed model consist of similarity measures is performed between the processing images. This type of measure is calculating the distance between the recovered image pixels with reference image pixel local indexing.

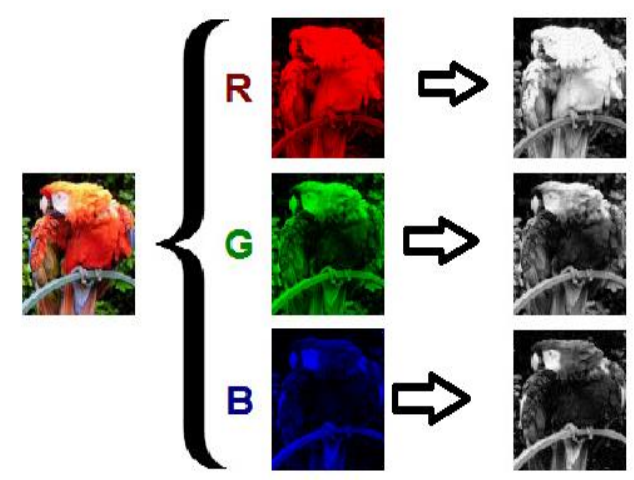

Figure 6 Conversion of RGB to Gray scale image representation

The maintaining the database for image retrieval is very essential for kalman estimating approach. This index is measured for set of retrieval images. The conversion of RGB to gray scale image representation is shown in Figure 6. Our query image details are distributing linearly through the fine tuning parameter. The intensity level of RGB is showing in figure 7.

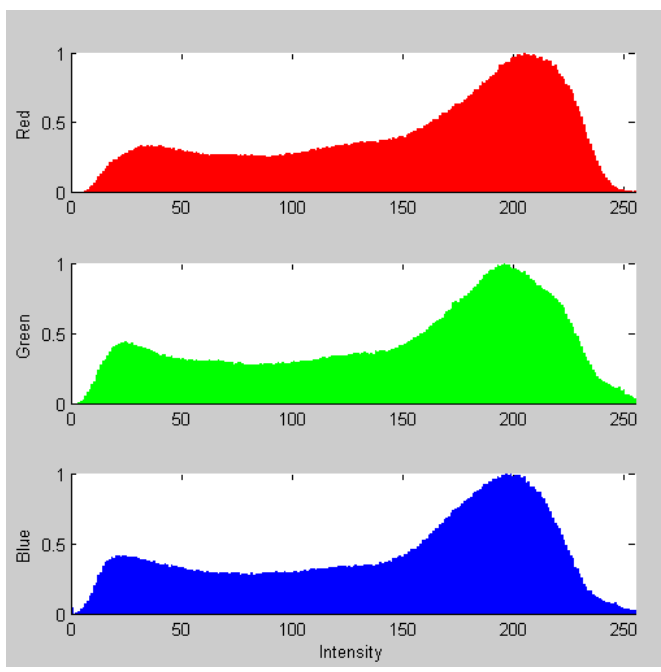

Figure 7 The intensity level of query image details 
Journal of Innovative Image Processing (JIIP) (2021)

Vol.03/ No. 01

Pages: 7-20

https://www.irojournals.com/iroiip/

DOI: https://doi.org/10.36548/jiip.2021.1.002

The overall proposed framework performance chart is compared with existing methods and it is proved superior. Figure 8 is showing the graph details of performance measure. Accuracy of our proposed model is very high due to adaptive state space framework construction. The reliability of our proposed work provides high compared to other method. But our computation time is suffering due to lot of equation solving.

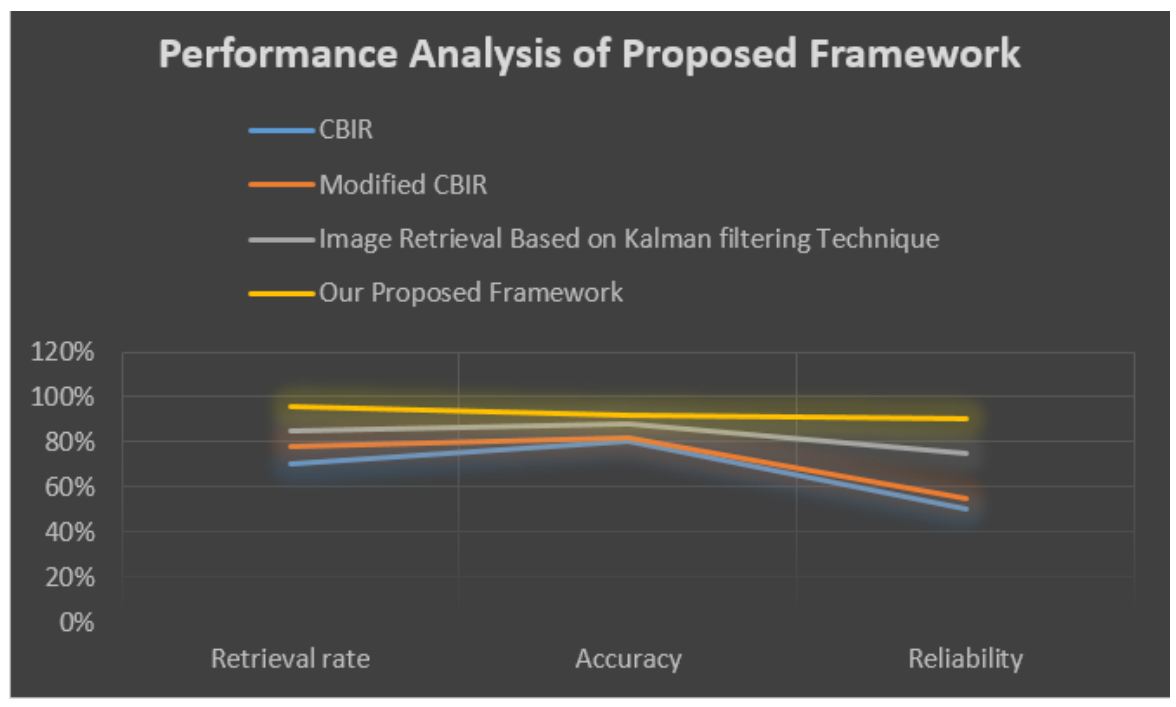

Figure 8 Performance comparison chart of framework

\section{CONCLUSION}

Thus, our new mechanism to image retrieval application by fine tuning adjusting of image intensity level of feature parameter. Our research work has focused to retrieve the images by adaptive state transition kalman filter approach. By updating estimation parameter for feature extraction database provided good results for our proposed framework. This observation is computing cross correlation between the images to ensemble the similarities measure. Initially, performed with filtering technique for content based image retrieval based on modified estimation parameter of state space model. The feature extraction includes RGB and gray level features of the image. Our proposed framework is very high of computation time compared to existing method due to large machine computation. In future we are going to concentrate on execution time. To reduce the computation time, the complexity of the process will be reduced by making standard equation to execute. Author wants to implement in this research work by applying machine 
Journal of Innovative Image Processing (JIIP) (2021)

Vol.03/ No. 01

Pages: 7-20

https://www.irojournals.com/iroiip/

DOI: https://doi.org/10.36548/jiip.2021.1.002

learning approach to reduce and execution time. The deep learning approach can provide more accurate results in image retrieval problem.

\section{REFERENCES}

[1] Y. Wei, Y. Zhao, C. Lu et al., "Cross-modal retrieval with CNN visual features: a new baseline," IEEE Transactions on Cybernetics, vol. 47, no. 2, pp. 449-460, 2017.

[2] P. Liu, J.-M. Guo, C.-Y. Wu, and D. Cai, "Fusion of deep learning and compressed domain features for content-based image retrieval," IEEE Transactions on Image Processing, vol. 26, no. 12, pp. 5706-5717, 2017.

[3] S. Yu, D. Niu, L. Zhang, M. Liu, and X. Zhao, "Colour image retrieval based on the hypergraph combined with a weighted adjacent structure," IET Computer Vision, vol. 12, no. 5, pp. 563-569, 2018.

[4] B.-H. Yuan and G.-H. Liu, "Image retrieval based on gradientstructures histogram," Neural Computing and Applications, 2019.

[5] R. C. Gonzalez and R. E. Woods, Digital Image Processing, Prentice-Hall, Upper Saddle River, NJ, USA, 3rd edition, 2007.

[6] D. H. Hubel and T. N. Wiesel, "Receptive fields of single neurones in the cat's striate cortex," \%e Journal of Physiology, vol. 148, no. 3, pp. 574-591, 1959.

[7] D. H. Hubel and T. N. Wiesel, "Receptive fields, binocular interaction and functional architecture in the cat's visual cortex," \%e Journal of Physiology, vol. 160, no. 1, pp. 106-154, 1962.

[8] S. Zeng, R. Huang, H. Wang, and Z. Kang, "Image retrieval using spatiograms of colors quantized by Gaussian Mixture Models,” Neurocomputing, vol. 171, pp. 673-684, 2016.

[9] A. Raza, H. Dawood, H. Dawood, S. Shabbir, R. Mehboob, and A. Banjar, "Correlated primary visual texton histogram features for content base image retrieval,” IEEE Access, vol. 6, pp. 4659546616, 2018.

[10] A. Raza, T. Nawaz, H. Dawood, and H. Dawood, "Square texton histogram features for image retrieval,” Multimedia Tools and Applications, vol. 78, no. 3, pp. 2719-2746, 2018.

[11] M. Verma, B. Raman, and S. Murala, "Local extrema cooccurrence pattern for color and texture image retrieval,” Neurocomputing, vol. 165, pp. 255-269, 2015.

ISSN: 2582-4252 (online) 
Journal of Innovative Image Processing (JIIP) (2021)

Vol.03/ No. 01

Pages: 7-20

https://www.irojournals.com/iroiip/

DOI: https://doi.org/10.36548/jiip.2021.1.002

[12] E. Walia, S. Vesal, and A. Pal, "An effective and fast hybrid framework for color image retrieval," Sensing and Imaging, vol. 15, no. 1, p. 93, 2014.

[13] J.-x. Zhou, X.-d. Liu, T.-w. Xu, J.-h. Gan, and W.-q. Liu, “A new fusion approach for content based image retrieval with color histogram and local directional pattern," International Journal of Machine Learning and Cybernetics, vol. 9, no. 4, pp. 677-689, 2018.

[14] S.-F. Chang, T. Sikora, and A. Purl, "Overview of the MPEG-7 standard," IEEE Transactions on Circuits and Systems for Video Technology, vol. 11, no. 6, pp. 688-695, 2001.

[15] Sathesh et al "A Dual Tree Complex Wavelet Transform Construction and its application to Image Denoising” International Journal of Image Processing (IJIP) Volume(3), Issue(6).

[16] Y. Ke and R. Sukthankar, "PCA-SIFT: A more distinctive representation for local image descriptors," in Proceedings of the 2004 IEEE Computer Society Conference on Computer Vision and Pattern Recognition, 2004 (CVPR 2004), Washington, DC, USA, June 2004.

[17] F. W. Campbell and J. J. Kulikowski, "Orientational selectivity of the human visual system," \%e Journal of Physiology, vol. 187, no. 2, pp. 437-445, 1966.

[18] F. Liu, H. Duan, and Y. Deng, "A chaotic quantum-behaved particle swarm optimization based on lateral inhibition for image matching," Optik, vol. 123, no. 21, pp. 1955-1960, 2012.

[19] J. Huang, S. R. Kumar, M. Mitra, W.-J. Zhu, and R. Zabih, "Image indexing using color correlograms," in Proceedings of IEEE Computer Society Conference on Computer Vision and Pattern Recognition, pp. 762-768, San Juan, Puerto Rico, June 1997.

[20] G. Pass, R. Zabih, and J. Miller, "Comparing images using color coherence vectors," in Proceedings of the Fourth ACM International Conference on Multimedia-MULTIMEDIA'96, pp. 65-73, New York, NY, USA, June 1997.

[21] W. Y. Ma and B. S. Manjunath, "A comparison of wavelet transform features for texture image annotation," in Proceedings of the International Conference on Image Processing, vol. 2, pp. 256-259, Washington, DC, USA, October 1995.

[22] W. Y. Kim and Y. S. Kim, “A region-based shape descriptor using Zernike moments,” Signal Processing: Image Communication, vol. 16, no. 1-2, pp. 95-102, 2000.

[23] G.-H. Liu and J.-Y. Yang, "Content-based image retrieval using color difference histogram," Pattern Recognition, vol. 46, no. 1, pp. 188-198, 2013. 
Journal of Innovative Image Processing (JIIP) (2021)

Vol.03/ No. 01

Pages: 7-20

https://www.irojournals.com/iroiip/

DOI: https://doi.org/10.36548/jiip.2021.1.002

[24] A. Chadha and Y. Andreopoulos, "Voronoi-based compact image descriptors: efficient region-of-interest retrieval with VLAD and deep-learning-based descriptors," IEEE Transactions on Multimedia, vol. 19, no. 7, pp. 1596-1608, 2017.

[25] N. Shrivastava and V. Tyagi, "An efficient technique for retrieval of color images in large databases," Computers \& Electrical Engineering, vol. 46, pp. 314-327, 2015.

[26] N. Varish, J. Pradhan, and A. K. Pal, "Image retrieval based on non-uniform bins of color histogram and dual tree complex wavelet transform," Multimedia Tools and Applications, vol. 76, no. 14, pp. 15885-15921, 2017.

[27] L. K. Pavithra and T. S. Sharmila, "An efficient framework for image retrieval using color, texture and edge features," Computers \& Electrical Engineering, vol. 70, pp. 580-593, 2018.

[28] J. Ahmad, M. Sajjad, I. Mehmood, S. Rho, and S. W. Baik, "Saliency-weighted graphs for efficient visual content description and their applications in real-time image retrieval systems," Journal of Real-Time Image Processing, vol. 13, no. 3, pp. 431-447, 2017.

[29] J. Ahmad, M. Sajjad, I. Mehmood, and S. W. Baik, "SSH: salient structures histogram for content based image retrieval," in Proceedings of the 2015 18th International Conference on Network-Based Information Systems (NBiS), pp. 212-217, Taipei, Taiwan, September 2015.

[30] J. Pradhan, A. K. Pal, and H. Banka, "Principal texture direction-based block level image reordering and use of color edge features for application of object based image retrieval," Multimedia Tools and Applications, vol. 78, no. 2, pp. 1685-1717, 2018.

[31] G.-H. Liu and J.-Y. Yang, "Exploiting color volume and color difference for salient region detection,” IEEE Transactions on Image Processing, vol. 28, no. 1, pp. 6-16, 2019.

[32] G.-H. Liu, "Content-based image retrieval based on Cauchy density function histogram," in Proceedings of the 12th International Conference on Natural Computation, Fuzzy Systems and Knowledge Discovery, pp. 506-510, Changsha, China, August 2016.

[33] G.-H. Liu, "Content-based image retrieval based on visual attention and the conditional probability," in Proceedings of the International Conference on Chemical, Material, and Food Engineering, pp. 838-842, Kunming, Yunnan, China, July 2015.

ISSN: 2582-4252 (online) 
Journal of Innovative Image Processing (JIIP) (2021)

Vol.03/ No. 01

Pages: 7-20

https://www.irojournals.com/iroiip/

DOI: https://doi.org/10.36548/jiip.2021.1.002

[34] J.-Z. Hua, G.-H. Liu, and S.-X. Song, "Content-based image retrieval using color volume histograms," International Journal of Pattern Recognition and Artificial Intelligence, vol. 33, no. 9, Article ID 1940010, 2019.

[35] J. Wu, L. Feng, S. Liu, and M. Sun, "Image retrieval framework based on texton uniform descriptor and modified manifold ranking," Journal of Visual Communication and Image Representation, vol. 49, pp. 78-88, 2017.

[36] S. Liu, J. Wu, L. Feng et al., "Perceptual uniform descriptor and ranking on manifold for image retrieval,” Information Sciences, vol. 424, pp. 235-249, 2018.

[37] Herráez J. and Ferri J., "Combining Similarity Measures in Content-Based Image Retrieval," Pattern Recognition Letters, vol. 29, no. 16, pp. 2174-2181, 2008.

[38] S. G. Narasimhan and S. K. Nayar, "Interactive Deweathering of An Image Using Physical Model," in IEEE Workshop on Color and Photometric Methods in Computer Vision, 2003.

[39] Jayaprabha P. and Somasundaram M., "Content Based Image Retrieval Methods using Graphical Image Retrieval Algorithm," Computer Science and Application, vol. 1, no. 1. pp. 9-14, 2012.

[40] N. Hautiere and D. Aubert, "Contrast Restoration of Foggy Images through use of an Onboard Camera,” in Proc. IEEE Conf. Intelligent Transportation Systems, pp. 601-606, Sep. 2005.

[41] T. Hiramatsu, T. Ogawa, and M. Haseyama, “A Kalman Filter based Restoration Method for In-vehicle Camera Images in Foggy Conditions," in Proc. IEEE Int. Conf. Acoustics, Speech, and Signal Processing (ICASSP 2008), pp. 1245-1248, Apr. 2008. 